\title{
Modelo de medición de variables de activos especializados en redes de producción
}

\author{
Cándida Santiago Ponce \\ Universidad Autónoma del \\ Estado de México \\ edu_marcan@yahoo.com \\ Francisco Ballina Ríos \\ Facultad de Contaduría y \\ Administración, UNAM \\ fballina@fca.unam.mx
}

\section{Resumen}

El propósito fundamental de este trabajo es medir la relación entre la especialización de los activos específicos con el desempeño en redes de producción; para ello se eligió la empresa automotriz Chrysler-Toluca y su red de proveedores como objeto de estudio. En esta medición se utilizaron siete variables (ubicación, activos físicos, activos humanos específicos, calidad, rapidez, costo de inventario y rentabilidad) derivadas de un soporte teórico (Williamson, 1985) y empírico (Dyer y Ouchi, 1993, entre otros), que fueron la base para la elaboración del cuestionario aplicado a 40 proveedores de autopartes de la cadena productiva para analizar la correlación de importancia que le dan estas empresas a esta integración. Los resultados de los cuestionarios se procesaron mediante el paquete estadístico SPSS; el estudio fue de corte transversal y no experimental para lo cual se utilizaron métodos complementarios como el panel de expertos dirigido a proveedores, así como la evolución de la empresa en una situación ya existente que garantizó la confiabilidad de la información.

Palabras clave: empresas ensambladoras, redes de proveedores, ventajas competitivas, especialización interempresarial. 


\title{
Measurement model for asset specificity and performance variables in supplier networks
}

\begin{abstract}
The objective of this research is to propose a measurement model of specific assets variables in the integration of Chrysler TAP (Toluca Assembly Plant) and its supplier networks case study. The central hypothesis to consider in this research is: "The Competitive Advantage of Chrysler Toluca enterprise" and "The specialization degree of their specific assets": Physical, human, location and performance in the production networks and their suppliers. The variables selection was founded on a theoretical support (Williamson, 1985) and an empirical support (Dyer and Ouchi, 1993), and others. This support served for the elaboration of a survey applied in the empirical research to a sample of 40 supplier companies of Chrysler's production network.
\end{abstract}

Keywords: autoindustry, supplier, networks, assets specificity, competitive advantage.

\section{Introducción}

El proceso de globalización de las grandes empresas ensambladoras (Ford, General Motors y Chrysler) y la reestructuración internacional de los proveedores de esta industria a menudo son tratados separadamente debido a la complejidad de ambos campos de estudio. Con las reglas del Tratado de Libre Comercio NAFTA, estas grandes empresas tomaron ventajas directas en México porque todas las autopartes que venían de la región norte de América eran consideradas como partes del contenido regional; la Volkswagen y Nissan tuvieron más problemas debido a sus lazos con proveedores de Alemania y Japón.

Este proceso de transformación de las relaciones productivas de las empresas automotrices fue crucial y mutuamente dependiente en un contexto de alta estandarización. En el nivel de ensamble significó un cambio de modelo que causó un complejo mecanismo de empuje a los proveedores, quienes si querían mantenerse en el mercado como proveedores directos, tenían que ofrecer una garantía de clase mundial a nivel consorcio. El doble desafío fue el proceso de concentración de capital y de internacionalización de la industria de proveedores en sus relaciones con compañías globales ensambladoras en una clara jerarquía globalizada definida por la relación de proveedores y ensambladores, a la vez en un eje local y global. 
Cabe aclarar que la productividad ganada en estas cadenas de valor sucede cuando las empresas pueden tener una ventaja competitiva sobre sus competidores, quienes también especializan sus inversiones; sugiere la creación de valor y de activos especializados no fácilmente imitados como desafío fundamental entre las empresas; asimismo, implica la inversión de activos básicos que deben identificar y medir para lograr una alta rentabilidad conjunta en redes de producción. De ahí que el propósito fundamental del presente estudio fue examinar la relación entre la especialización de los activos y el desempeño en la integración de redes de producción de estos transactores con un alto grado de especialización interempresarial que incrementa su desempeño; para llevar a cabo esto, se eligió la empresa automotriz Chrysler, Toluca y sus proveedores de autopartes por ser una empresa transnacional a la vanguardia en tecnología.

\section{Antecedentes y planteamiento del problema}

La importancia de la industria maquiladora de autopartes en el nivel nacional radica en que es la principal fuente de divisas para el país después del petróleo; de igual manera, ha llegado a representar más de una tercera parte de las exportaciones totales de México y es fuente generadora de empleos con un personal ocupado de 258 mil 895 trabajadores. De acuerdo con cifras proporcionadas por la Sedeco del gobierno del Estado de México, la industria automotriz de la entidad exporta más vehículos que cualquiera de los países de América Latina, con excepción de Brasil.

Hoy en día la industria automotriz es una industria madura que presenta problemas como son mercados saturados en los países desarrollados, exceso de capacidad instalada, altos grados de segmentación y proliferación de productos, cerrada competencia en precios y márgenes de utilidad decreciente. Las empresas automotrices ensambladoras controladas por corporaciones multinacionales ubicadas fuera del país de origen son un "integrador de sistemas" (INEGI, 2006, p. 54) porque los proveedores diseñan, desarrollan e instalan módulos de componentes (Shenkar, 2004, p. 88).

Esto nos conduce a plantear la problemática y analizarla a partir de hechos y explicaciones de diversos autores de la teoría administrativa y organizacional para fundamentarla con argumentos científicos y metodológicos. 


\section{Marco teórico}

El cambiante contexto mundial ha tenido repercusiones sobre las economías locales y regionales. La integración mundial y las presiones de la competitividad internacional se están intensificando en un momento en que se perciben como transitorias las ventajas competitivas tradicionales. La globalización de actividades industriales ha provocado intensos debates que han contribuido a popularizar conceptos y cuerpos analíticos como la economía de costos de transacción, asociados con conceptos más administrativos como las cadenas de valor (Porter, 1980, 1985) y las alianzas estratégicas (Banville, Chanaron, 1991; Baudry, 1995). Estos conceptos microeconómicos impregnan hoy en día la ciencia de la gestión.

A las ensambladoras y proveedores les interesa cooperar entre sí, pero también apropiarse de los beneficios económicos y tecnológicos derivados del intercambio de información de las mismas. La cooperación implica conflicto, toda vez que las empresas intentan maximizar ingresos al mismo tiempo que coevolucionan con otras. En estos conglomerados (clusters) - la ubicación geográfica de las empresas, proveedores especializados, instituciones asociadas, universidades, organismos normativos, asociaciones gremiales - compiten, pero también cooperan; en esta perspectiva Porter (1991) expone la teoría de la competitividad.

La teoría económica evolutiva señala que las empresas deben aprender a sobrevivir y crecer apropiándose de los recursos del entorno e internacionalizándolos de manera eficiente (selección natural darwiniana), pero para aprender a adaptarse a las variaciones de un ambiente adverso, incierto y complejo (aprendizaje lamarkiano) necesitan cooperar en redes de empresas (aprendizaje vía coevolución) (Porter, 1991).

Lundvall (1985) considera que el elemento determinante que impulsa a proveedores y usuarios a intercambiar información cualitativa y a cooperar de manera directa es la unidad entre las oportunidades tecnológicas; sin embargo, este autor no deja de señalar que en la relación aparte de los vínculos de cooperación y los intercambios de información cualitativa hay elementos de poder y dominio de lealtad y confianza.

En las concepciones sobre la relación proveedor-usuario es menester proporcionar una taxonomía de las teorías de la empresa. En cada enfoque de dicha relación subyace sin duda una idea particular de lo que es la empresa con base en los trabajos de Sidney (1993). En el cuadro 1, en la matriz de dos por dos, sobre las columnas, 
se distinguen dos focos de atención de acuerdo con la que cada teoría a su vez le ha dado: producción frente a intercambio.

En los siguientes renglones se presenta el binomio de supuestos de racionalidad ilimitada frente a racionalidad limitada. Si el análisis se centra en la producción de la empresa y se considera esta toma de decisiones a partir del supuesto de racionalidad ilimitada, entonces se ubica en el primer cuadrante; correspondiente a la teoría neoclásica ortodoxa, como se presenta en los manuales de economía.

En el cuadrante formado por la intersección de racionalidad limitada y producción se puede situar a la teoría económica evolutiva, ésta — como se explicó— centra su análisis en la especificidad de la tecnología utilizada, las habilidades de aprendizaje y las rutinas organizacionales (ver cuadro 1).

\section{Cuadro 1}

\section{Paradigmas contemporáneos en la teoría de la empresa}

\begin{tabular}{l|lll}
\hline \multirow{2}{*}{$\begin{array}{l}\text { Racionalidad } \\
\text { ilimitada }\end{array}$} & $\begin{array}{l}\text { Troducción } \\
\text { (trayectoria tecnológica) }\end{array}$ & \multicolumn{1}{c}{ Intercambio } \\
\cline { 2 - 4 } & $\begin{array}{l}\text { Teoría } \\
\text { neoclásica }\end{array}$ & $\begin{array}{l}\text { Teoría de la ortodoxia } \\
\text { de los cuadernos de trabajo. } \\
\text { (Working Paper orthodoxy) }\end{array}$ \\
$\begin{array}{l}\text { Racionalidad } \\
\text { limitada }\end{array}$ & $\begin{array}{l}\text { Teoría } \\
\text { económica } \\
\text { evolutiva } \\
\text { (Nelson, Winter y Dosi) }\end{array}$ & $\begin{array}{l}\text { B. A. Lundvall } \\
\text { (Mercados organizados) }\end{array}$ & $\begin{array}{l}\text { Teoría de los costos } \\
\text { de transacción } \\
\text { (Coase, Williamson yNorth) }\end{array}$ \\
\hline
\end{tabular}

Fuente: Winter (1993)

Estas determinantes gobiernan tanto el desempeño productivo como las capacidades para innovar y relacionarse con otros agentes (clientes, proveedores, etc.). La teoría evolutiva, lo mismo que la neoclásica ortodoxa, no tiene al intercambio como eje de análisis de la empresa sino a su desempeño interno; por eso, ambas se ubican en la misma columna.

La teoría de los costos de transacción supone una racionalidad limitada y su centro de análisis es el intercambio, es decir, las relaciones contractuales entre unidades 
productivas autónomas. Este enfoque parte de la idea de que hay diferentes formas de organizar las transacciones entre las empresas, lo sobresaliente es el intento de vincular la organización con las transacciones y los costos. Existe, además, una vertiente teórica cercana a la teoría ortodoxa, que es la de Sidney (1993) quien la ha denominado working paper orthodoxy. Se trata de estudios teóricos que analizan a las empresas conservando el supuesto de racionalidad ilimitada, pero que se interesan también en los problemas del intercambio; no se preocupan tanto de la producción como de la estructura de las relaciones entre los actores involucrados, su análisis incluye el problema de los incentivos, el control y la información que fluye entre los propietarios de la empresa, gerentes y de éstos con los trabajadores.

Las investigaciones respectivas se enmarcan en modelos de equilibrio no cooperativos, teoría de grupos o análisis del segundo óptimo. Existe un enfoque teórico que no podría clasificarse en ninguno de los cuadrantes de la taxonomía, se trata del concepto de mercados organizados de Lundvall (1985), que integra elementos de la producción y el intercambio. Este modelo supone una racionalidad limitada y el intercambio de información cualitativa entre empresas, pero la información, el aprendizaje interactivo y la trayectoria tecnológica aparecen como variables fundamentales en vez de los costos de transacción.

\section{La empresa automotriz Chrysler de Toluca}

El sector automotriz en el Estado de México es considerado como el de mayor intensidad económica gracias a la actividad de firmas como Chrysler, General Motors, BMW, Mercedes Benz y Ford Motor Company, entre muchas del ramo que se desarrollan desde hace varias décadas en la entidad, con una aportación del $26 \%$ del PIB manufacturero de 6 mil 170 millones.

La fábrica Auto-Mex, mejor conocida como Chrysler, fue fundada en 1939 por un grupo de hombres de negocios encabezados por Gastón Azcárraga Vidaurreta con la intención de armar los productos de dicha marca provenientes de Estados Unidos. La planta inaugurada en aquel entonces ocupó un área total construida de 8.169 metros cuadrados y tenía capacidad para producir 120 unidades mensuales. Para 1960, esta planta ocupaba ya una superficie construida de 64183 metros cuadrados y producía 1200 unidades al mes, teniendo una capacidad de armado de 1 870 autos. Debido a su alto nivel de calidad, esta planta fue seleccionada en 2005 
para fabricar el popular Chrysler PT Cruiser y exportarlo a 60 países hasta el 9 de julio del $2010 .^{1}$

A finales del año 2007 la empresa Daimler se fusionó con la empresa Chrysler, contando con un total de 406 proveedores de autopartes de 58 componentes. En el año 2008 se ensamblaban por día 878 unidades y se contaba con 300 personas por turno, produciendo una unidad cada 1.64 minutos y fracción en promedio; asimismo, se contaba con un inventario máximo de 3 días a corto plazo y a mediano plazo con un margen de mes y medio, teniendo una respuesta de diseño cada dos años. Con una inversión de mil millones de pesos en ese año, la empresa adquiere 350 robots, ya que anteriormente contaba con 150 .

En el año 2008 la industria automotriz cerró con despidos de aproximadamente 600 mil trabajadores en las plantas Ford, Chrysler y G.M., lo cual se debió a la disminución de importaciones provenientes de Estados Unidos, quien le compró a México el $70 \%$ de los autos que producía. A finales de este año, la empresa Daimler se retira del grupo Chrysler, Dodge y Jeep, como consecuencia en la caída de sus ventas del $19.8 \%$. En el año 2009 la producción nacional de vehículos cayó en un 39.4\% como consecuencia de la severa crisis mundial que repercutió en México, siendo la planta Toluca una de las más afectadas con similares circunstancias en el norte y centro del país, por este suceso la planta Chrysler-Toluca, cerró el primero de mayo del 2009 reanudando actividades el 3 de agosto del mismo año.

Hoy en día la empresa Chrysler, Toluca, cuenta con dos plantas: una de ensamble y otra de estampado; la primera con una superficie de 1 millón 601611 metros cuadrados, en la que a partir del 13 de diciembre de 2009 se empezó a fabricar el Dodge Journey y contaba con las siguientes líneas de producción: carrocería, ensamble y pintura; actualmente, esta planta para la fabricación de este modelo compra 2498 autopartes, por lo que cuenta con un total de 685 proveedores, de los cuales 275 son nacionales, 297 extranjeros y 113 locales. La capacidad de producción actual de esta planta es de un promedio de 640 a 700 unidades diarias del modelo Dodge/ Journey; se ensambla una unidad por minuto y 58 a 60 unidades en promedio por hora para satisfacer principalmente la demanda del mercado de Estados Unidos.

${ }^{1}$ Departamento de Ingeniería Industrial, Chrysler, Toluca. 
El 31 de agosto de 2011 se iniciaron los trabajos para montar la línea de ensamble del vehículo subcompacto FIAT 500-241, el primero de la alianza Fiat-Chrysler con una nueva cadena de proveedores, componentes, autopartes, realizando pruebas de simulación para empezar a producir 80 unidades por semana a partir del mes de febrero de 2011 para la exportación principalmente a los mercados de Estados Unidos, Europa y América Latina. ${ }^{2}$

Esta planta cuenta con una inversión aproximada de \$211000 millones de USD en activos físicos como maquinaria y equipo, considerando que tienen en esta planta un total de 422 robots para la fabricación de las unidades Dodge Journey y el Fiat 500-241, además de que el costo de inversión de cada robot es de $\$ 500000$ dólares americanos. La planta cuenta con un total de 2630 trabajadores y 229 empleados.

La planta de estampado fue fundada en marzo de 1994 con una superficie de 11 400 metros cuadrados; produce paneles de carrocería para la Dodge Journey y el Fiat 500; tiene un total de 173 trabajadores y 30 empleados; fue la primera planta de Chrysler Grupo en obtener el certificado de mantenimiento productivo total desde octubre de 2005; además, sus funcionales estructuras se distinguen por el uso de tecnología de punta y un alto índice de productividad. El interior del complejo está dedicado a la recepción, administración, control de inventario, despacho y distribución de refacciones Mopar, así como a las refacciones para los distribuidores de la marca Mercedes Benz. En el siguiente cuadro 2 se presenta el volumen de producción total en el periodo comprendido del 2006 al 2010.

\section{Cuadro 2}

Producción total para exportación y nacional de la empresa automotriz Chrysler, planta Toluca 2006-2010

\begin{tabular}{c|r|r|r|c|c}
\hline Año & $\mathbf{2 0 0 6}$ & $\mathbf{2 0 0 7}$ & $\mathbf{2 0 0 8}$ & $\mathbf{2 0 0 9}$ & $\mathbf{2 0 1 0}$ \\
\hline $\begin{array}{c}\text { Total exportación } \\
\text { Total nacional }\end{array}$ & $\begin{array}{r}169475 \\
4780\end{array}$ & $\begin{array}{r}112974 \\
1906\end{array}$ & $\begin{array}{r}168103 \\
14548\end{array}$ & $\begin{array}{r}71202 \\
12144\end{array}$ & $\begin{array}{c}164894 \\
18291\end{array}$ \\
\hline Producción total & 174255 & 114880 & 182651 & 83346 & 183185 \\
\hline
\end{tabular}

Fuente: Datos históricos de Chrysler, planta Toluca 2005 a $1^{\circ}$ de octubre de 2010. El cuadro incluye la producción total destinada a los mercados nacional e internacional.

${ }^{2}$ Departamento de Ingeniería Industrial, Chrysler, Toluca. 
Asimismo, en este cuadro 2 se observa dos momentos coyunturales en la producción total de la empresa automotriz Chrysler, planta Toluca; por un lado, hay una disminución del 65.92\% de su producción total en 2007 con respecto al año anterior y nuevamente una disminución del $45.63 \%$ de su producción total en 2009 con respecto del año anterior; por otro, un aumento del $54.48 \%$ en su producción total en 2010; en este mismo año, el $69.2 \%$ de la producción total para exportación se envió a Estados Unidos (114 106 unidades), el 8.1\% a Canadá (13 356 unidades), el 9.8\% a Europa (16 159 unidades), el 10.1\% a Latinoamérica (16 654 unidades), el $2.1 \%$ a Asia (3 464 unidades) y en este mismo año África compró el $0.7 \%$ del total exportado (1 154 unidades) (AMIA, 2010).

\section{Selección de variables y diseño del cuestionario}

La selección de variables para proponer un modelo de medición sobre la integración de redes de producción de la empresa automotriz Chrysler, planta Toluca, con sus proveedores se realizó a través de un soporte teórico y empírico fundamentado por diversos autores (Alchain, 1972; Klein, Crawford y Alchain, 1978; Asanuma, 1989; North, 1990; Schoemaker y Amit, 1994; Dyer y Ouchi, 1996 y Williamson, 1985), quienes argumentan que el desempeño se realiza a través de las inversiones en activos especializados.

\section{Los activos específicos y el desempeño}

Si una empresa no convierte sus activos generales (trabajo, capital y tecnología) en activos especializados pierde el valor agregado; de esta forma, como Sako (1992, p. 26) observa que la "interfirma" con eficiencia X se refiere a un par de socios que están juntos con esta coespecialización o relación de activos especializados, los socios podrán generar relaciones de cuasirenta (Aoki, 1988).

\section{Los activos específicos y la calidad}

Clark y Fujimoto (1991) estudiaron cómo se incrementa la calidad cuando hay menos variación en los procesos organizacionales y pocos proveedores, así como se da un incremento en la rapidez de retroalimentación y realización de datos, ambos a través de empresas en la producción de redes.

Estudios previos sugirieron que una efectiva coordinación en el diseño y manufactura pueden mejorar la calidad. En particular cuando los proveedores y los 
ingenieros compradores desarrollan relaciones específicas de know how y tienen experiencia substancial trabajando juntos; por lo tanto, la coespecialización de los recursos humanos interempresa está relacionada con una alta calidad del producto.

\section{Los activos específicos y el nuevo modelo en el ciclo del tiempo}

La habilidad de desarrollar nuevos productos rápidamente fue un importante recurso de ventaja competitiva en muchas industrias. Históricamente las ventas de carros se incrementaron substancialmente en Estado Unidos y Japón en las empresas automotrices que podían desarrollar un nuevo modelo más rápido que sus competidores porque sus modelos eran más avanzados con lo último en tecnología Takahiro (2001). Williamson (1985) ha argumentado que los proveedores ganan en un contrato inicial y tendrán ventaja en el próximo contrato debido al aprendizaje que incluye la propiedad técnica, procedimientos administrativos y habilidades en tareas específicas.

A partir de entonces se ha tenido una mayor habilidad de las empresas automotrices de coordinar el diseño y manufactura con sus proveedores. Las empresas automotrices han usado estandarización de partes con una alta calidad percibida por los consumidores como un producto total que es el vehículo conforme a las expectativas del cliente (Takahiro, 2001).

\section{Los activos físicos específicos y los costos de inventarios}

Se ha sugerido que los transactores tienen inversiones en una ubicación específica con la finalidad de economizar el manejo del inventario y los costos de transportación deben tener bajos stocks y bajos costos de inventario (Williamson, 1985). 


\section{Cuadro 3 \\ Modelo de relación de variables de activos especializados y la integración de redes de producción}

\begin{tabular}{|c|c|c|c|c|}
\hline $\begin{array}{l}\text { Variable y definición } \\
\text { conceptual }\end{array}$ & $\begin{array}{r}\text { Definición } \\
\text { operacional }\end{array}$ & Indicador & $\begin{array}{c}\text { Dimensión } \\
\text { (escalaLikent) }\end{array}$ & Ítems \\
\hline $\begin{array}{l}\text { 1. Ubicación } \\
\text { específica: proximidad } \\
\text { de la empresa y sus } \\
\text { proveedores. }\end{array}$ & $\begin{array}{l}\text { Distancia en } \\
\text { kilómetros entre } \\
\text { la empresa y los } \\
\text { proveedores. }\end{array}$ & $\begin{array}{l}\text { Grado de } \\
\text { importancia. }\end{array}$ & 1 a 5 & $\begin{array}{l}\text { ¿Qué importancia le da a } \\
\text { la ubicación y acciones de } \\
\text { localización para un desarrollo } \\
\text { futuro de integración de redes de } \\
\text { producción con la empresa? }\end{array}$ \\
\hline $\begin{array}{l}\text { 2. Activos físicos } \\
\text { específicos: inversión } \\
\text { en capital y equipo (no } \\
\text { recuperable). }\end{array}$ & $\begin{array}{l}\text { Inversión de } \\
\text { capital y equipo } \\
\text { de la empresa en } \\
\text { sus proveedores. }\end{array}$ & $\begin{array}{l}\text { Grado de } \\
\text { importancia. }\end{array}$ & 1 a 5 & $\begin{array}{l}\text { ¿Qué importancia le da a las } \\
\text { inversiones en capital y equipo } \\
\text { (no recuperable) y acciones } \\
\text { para el desarrollo futuro de } \\
\text { la integración de redes de } \\
\text { producción con la empresa? }\end{array}$ \\
\hline $\begin{array}{l}\text { 3. Activos humanos } \\
\text { específicos: } \\
\text { conocimiento acumulado } \\
\text { a través del tiempo } \\
\text { en relaciones de } \\
\text { experiencia. }\end{array}$ & $\begin{array}{l}\text { Asistencia y } \\
\text { colaboración que } \\
\text { dan los ingenieros } \\
\text { de la empresa a } \\
\text { sus proveedores. }\end{array}$ & $\begin{array}{l}\text { Grado de } \\
\text { importancia. }\end{array}$ & 1 a 5 & $\begin{array}{l}\text { ¿Importancia que tiene para } \\
\text { el proveedor la asistencia } \\
\text { y colaboración que dan los } \\
\text { ingenieros de la empresa, y } \\
\text { acciones para el desarrollo } \\
\text { futuro de integración de redes de } \\
\text { producción? }\end{array}$ \\
\hline $\begin{array}{l}\text { 4. Calidad: cumplir } \\
\text { con las expectativas } \\
\text { del cliente en tiempo y } \\
\text { forma. }\end{array}$ & $\begin{array}{l}\text { Asistencia y } \\
\text { colaboración que } \\
\text { dan ingenieros a } \\
\text { las proveedoras. }\end{array}$ & $\begin{array}{l}\text { Grado de } \\
\text { importancia. }\end{array}$ & 1 a 5 & $\begin{array}{l}\text { ¿Qué importancia le da a la } \\
\text { asistencia y colaboración que } \\
\text { dan los ingenieros de la empresa } \\
\text { para cumplir expectativas y } \\
\text { acciones para el desarrollo } \\
\text { futuro de integración de redes de } \\
\text { producción? }\end{array}$ \\
\hline $\begin{array}{l}\text { 5. Rapidez: ajuste en } \\
\text { el desarrollo de nuevos } \\
\text { modelos el ciclo del } \\
\text { tiempo más rápido que } \\
\text { la competencia en una } \\
\text { coordinación efectiva }\end{array}$ & $\begin{array}{l}\text { Colaboración para } \\
\text { ajustarse rápido al } \\
\text { nuevo modelo. }\end{array}$ & $\begin{array}{l}\text { Grado de } \\
\text { importancia. }\end{array}$ & 1 a 5 & $\begin{array}{l}\text { ¿Qué importancia tiene el tiempo } \\
\text { de ajuste que da la empresa } \\
\text { cuando hace cambio de modelo } \\
\text { y las acciones para el desarrollo } \\
\text { futuro de integración de redes de } \\
\text { producción? }\end{array}$ \\
\hline $\begin{array}{l}\text { 6. Costo de inventario: } \\
\text { derivado de la } \\
\text { coordinación y ubicación } \\
\text { de proveedores. }\end{array}$ & $\begin{array}{l}\text { Colaboración y } \\
\text { colaboración entre } \\
\text { ensambladores } \\
\text { de la empresa y } \\
\text { proveedores. }\end{array}$ & $\begin{array}{l}\text { Grado de } \\
\text { importancia. }\end{array}$ & 1 a 5 & $\begin{array}{l}\text { ¿Qué importancia tienen la } \\
\text { colaboración y coordinación con } \\
\text { los ingenieros de la empresa para } \\
\text { minimizar costos de inventario } \\
\text { y acciones para el desarrollo } \\
\text { futuro de integración de redes de } \\
\text { producción? }\end{array}$ \\
\hline $\begin{array}{l}\text { 7. Rentabilidad: } \\
\text { beneficios para ambos } \\
\text { transactores. }\end{array}$ & $\begin{array}{l}\text { Beneficio para } \\
\text { la empresa y sus } \\
\text { proveedores. }\end{array}$ & $\begin{array}{l}\text { Grado de } \\
\text { importancia. }\end{array}$ & 1 a 5 & $\begin{array}{l}\text { ¿Qué importancia tiene la } \\
\text { rentabilidad y acciones para el } \\
\text { desarrollo futuro de integración } \\
\text { de redes de producción? }\end{array}$ \\
\hline
\end{tabular}

Fuente: El diseño del cuestionario forma parte de la propia investigación. El cuadro incluye la operatividad de las siete variables incluidas en el cuestionario 


\section{Los activos específicos y la rentabilidad}

La relación de inversiones especializadas incrementa la calidad, reduce el ciclo de tiempo de nuevos modelos y minimiza los costos de inventario; estos beneficios se transforman en altas ganancias. Consecuentemente se espera que las empresas automotrices y su grupo de proveedores obtengan más utilidades y, por ende, una alta rentabilidad. Finalmente, debemos notar que la ubicación específica, los activos físicos especializados y activos humanos incrementan la interacción de los recursos humanos (Enright, 1995). Éstas son las variables relevantes por considerar en el presente modelo de medición de los activos especializados y el desempeño en la integración de la empresa Chrysler y su red de proveedores, derivadas del modelo de Dyer (1996). La operatividad de las variables y elaboración del cuestionario forman parte de la presente investigación, la cual se presenta en el cuadro anterior.

\section{Investigación de campo}

Para la investigación de campo se aplicó un cuestionario, elaborado a partir de las variables antes mencionadas, a una muestra de 40 empresas proveedoras de autopartes de la empresa automotriz de estudio, la cual representó el 10\% (40 empresas proveedoras) de un total de 400 (año 2007), de los cuales el 20\% fueron proveedores locales, el $42.5 \%$ proveedores nacionales y el $37.5 \%$ proveedores extranjeros. El $57.5 \%$ de la muestra fue del giro automotriz, el $40 \%$ de autopartes y el $3.5 \%$ de manufactura.

En este estudio se consideró un nivel de significancia del .95 y un error del $0.5 \%$; fue de tipo no experimental, de corte transversal, utilizando métodos complementarios como el panel de expertos dirigido a los proveedores, así como la evolución de esta empresa en una situación ya existente, lo que garantizó la confiabilidad de la información y tuvo como propósito fundamental analizar la correlación de importancia que le dan las empresas proveedoras de autopartes a la integración de redes de producción, considerando las siete variables enunciadas. Para el diseño del cuestionario se utilizaron preguntas en escala de Likert con parámetros del 1 a 5 que van de nada importante, poco importante, algo importante, importante y muy importante.

El cuestionario se dividió en cuatro bloques: el primero para anotar datos específicos de cada empresa proveedora; el segundo con una batería de 13 preguntas para 
medir la importancia que tiene para cada una de estas empresas la especialización interfirma con la empresa automotriz Daimler-Chrysler, Toluca; el tercero con una serie de 11 preguntas para medir la importancia que le dan los proveedores a las acciones de desarrollo futuro para mejorar la integración de redes de producción con la empresa en estudio; y el cuarto en la que cada empresa proveedora enumeró los principales problemas por orden de importancia y explicó la repercusión que pueden tener estos problemas y sus propuestas de solución.

\section{Resultados de la investigación de campo}

Una vez obtenidos los resultados de los cuestionarios del departamento de compras de la empresa en estudio, se procedió a tabular y codificar los datos obtenidos mediante el paquete estadístico SPSS para lo que utilizó el coeficiente de correlación de Pearson para la comprobación de hipótesis, eligiendo las correlaciones más altas y menores de 1.00 con un error estimado de 0.05 y un nivel de significancia de .95. A continuación se presentan los resultados de la encuesta.

\section{Análisis estadístico de los datos}

Para el análisis estadístico de datos, en primer lugar, se obtuvieron las distribuciones de cada una de las siete variables de estudio, derivadas de la interpretación descriptiva que es favorable a la actitud de los proveedores hacia la importancia de la integración de redes de producción con la empresa automotriz de estudio; al describir nuestros datos interpretamos la mediana como medida de tendencia central y de la variabilidad en conjunto no aisladamente. Posteriormente, se utilizó el coeficiente de Pearson considerando que este coeficiente puede variar de $-1.00 \mathrm{a}+1.00$, donde -1.00 indica una correlación negativa perfecta y +1.00 indica una correlación positiva perfecta. De las siete variables, los proveedores hicieron énfasis en la variable rapidez en el desarrollo del nuevo modelo con una correlación positiva de 0.649 (ver cuadro 4). 


\section{Cuadro 4 \\ Grado de importancia que le dan los proveedores a las variables de integración de redes de producción}

\begin{tabular}{|c|c|c|}
\hline \multicolumn{2}{|l|}{ Variable totalmente importante } & $\begin{array}{l}\text { Grado de } \\
\text { correlación }\end{array}$ \\
\hline Rapidez en el desarrollo del nuevo producto & $\begin{array}{l}\text { Tiempo que da Daimler- } \\
\text { Chrysler a sus proveedores } \\
\text { en el cambio de nuevo } \\
\text { modelo }\end{array}$ & 0.649 \\
\hline \multicolumn{2}{|l|}{ Variable totalmente importante } & $\begin{array}{l}\text { Grado de } \\
\text { correlación }\end{array}$ \\
\hline \multicolumn{2}{|c|}{$\begin{array}{l}\text { Mejorar la localización de plantas proveedoras/tiempo que da Daimler } \\
\text { Chrysler ajustarse al cambio del nuevo modelo }\end{array}$} & 0.394 \\
\hline \multicolumn{2}{|c|}{ Variable totalmente importante } & $\begin{array}{l}\text { Grado de } \\
\text { correlación }\end{array}$ \\
\hline \multicolumn{2}{|c|}{$\begin{array}{l}\text { Mejorar las inversiones en capital y equipo (no recuperable)/ mejorar la } \\
\text { asistencia que dan ingenieros de Daimler Chrysler }\end{array}$} & 0.396 \\
\hline \multicolumn{2}{|l|}{ Variable totalmente importante } & $\begin{array}{l}\text { Grado de } \\
\text { correlación }\end{array}$ \\
\hline \multicolumn{2}{|c|}{$\begin{array}{l}\text { Mejorar la colaboración para compartir información confidencial en planes y } \\
\text { costos detallados de producción/contratos flexibles }\end{array}$} & 0.412 \\
\hline \multicolumn{2}{|l|}{ Variable totalmente importante } & $\begin{array}{l}\text { Grado de } \\
\text { correlación }\end{array}$ \\
\hline \multicolumn{2}{|c|}{$\begin{array}{l}\text { Cumplimiento de especificaciones normas ISO9000/mejorar asistencia que } \\
\text { dan ingenieros de Daimler Chrysler a proveedores. }\end{array}$} & 0.410 \\
\hline \multicolumn{2}{|c|}{ Variable totalmente importante } & $\begin{array}{l}\text { Grado de } \\
\text { correlación }\end{array}$ \\
\hline \multicolumn{2}{|c|}{$\begin{array}{l}\text { Tiempo suficiente que da Daimler Chrysler para que sus proveedores se ajusten a } \\
\text { nuevos modelos/contratos flexibles. }\end{array}$} & 0.413 \\
\hline \multicolumn{2}{|l|}{ Variable totalmente importante } & $\begin{array}{l}\text { Grado de } \\
\text { correlación }\end{array}$ \\
\hline \multicolumn{2}{|c|}{$\begin{array}{l}\text { Inventario de autopartes de proveedores/colaboración para compartir } \\
\text { información confidencial en planes y costos detallados de producción. }\end{array}$} & 0.401 \\
\hline \multicolumn{2}{|c|}{ Variable totalmente importante } & $\begin{array}{l}\text { Grado de } \\
\text { correlación }\end{array}$ \\
\hline \multicolumn{2}{|c|}{ Rentabilidad de Daimler Chrysler y proveedores/contratos flexibles. } & 0.376 \\
\hline \multicolumn{2}{|l|}{ Variable totalmente importante } & $\begin{array}{l}\text { Grado de } \\
\text { correlación }\end{array}$ \\
\hline \multicolumn{2}{|c|}{$\begin{array}{l}\text { Mejorar la colaboración para compartir información confidencial en costos } \\
\text { detallados de producción y contratos flexibles entre Daimler Chrysler y sus } \\
\text { proveedores. }\end{array}$} & 0.401 \\
\hline
\end{tabular}

Nota: En escala de $0.1=$ Nada importante a $1=$ Totalmente importante.

Fuente: Resultados de las correlaciones derivados del estudio. 
En la opinión de los proveedores existe una correlación estadísticamente significativa en las variables de los activos específicos y el desempeño en redes de producción; asimismo, proponen como principales medidas de acción las siguientes: mejorar aún más la comunicación, compartir información confidencial de planes y costos, efectuar economías de escala para reducir costos de producción, cumplir con los requerimientos justo a tiempo y respetar la rapidez en el desarrollo del nuevo modelo. A continuación se presentan en orden de importancia de las siete variables de estudio los resultados de la encuesta.

\section{Gráfica 1 \\ Importancia de la integración de redes de producción en Daimler-Chrysler de Toluca}

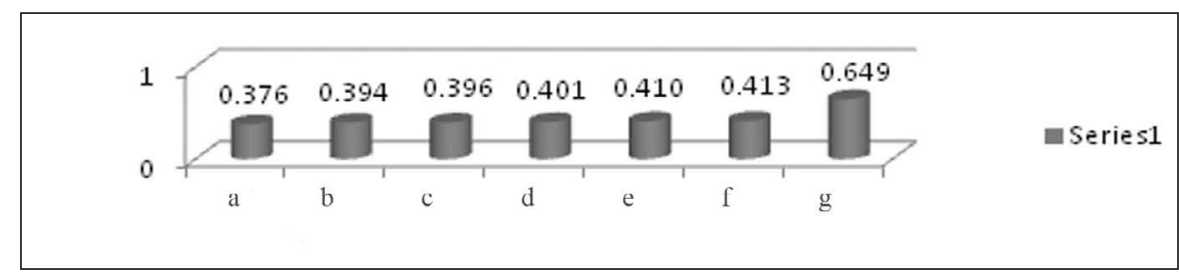

$\mathrm{a}=$ rentabilidad, $\mathrm{b}=$ localización de la planta, $\mathrm{c}=$ inversión en capital y equipo, d=inventario de autopoartes, $\mathrm{e}=$ complemento de norma ISO 9000, $\mathrm{f}=$ tiempo para ajustar nuevo modelo, $\mathrm{g}=$ rapidez en el desarrollo del nuevo producto

Fuente: Correlaciones de las variables de estudio para la comprobación de hipótesis establecidas en la investigación.

Una vez obtenidos los resultados de los encuestados la variable más importante de las siete fue la rapidez de tiempo de ajuste cuando hay cambio de un nuevo modelo; esto aunado a la importancia de los recursos físicos específicos y de la ubicación geográfica de lo cual resulta un cuarto nivel que es la rentabilidad (gráfica 1).

\section{Conclusiones}

La teoría de la racionalidad limitada, como la teoría de los costos de transacción, sustentan las variables fundamentales de las grandes empresas ensambladoras y sus proveedores: intercambio de información, relaciones contractuales autónomas, aprendizaje interactivo y trayectoria tecnológica compartida. 
El alcance de esta investigación fue el de validar la clasificación de los activos especializados de Williamson (1983 y 1985) y el modelo de redes de producción de Jeffrey H. Dyer (1995), así como su relación con variables de desempeño como recurso de ventaja competitiva y evidencia en la industria automotriz, derivado del caso de la empresa de estudio. Se confirma las hipótesis de investigación en relación con la integración de redes de producción para el logro de la eficiencia, lo cual deja un precedente para posteriores estudios longitudinales.

Las limitaciones fueron la disponibilidad de los datos, aunado a las políticas de la propia empresa Chrysler, Toluca, en cuanto a la disponibilidad de información confidencial. Otros aspectos que podrían ser investigados son las ventajas comparativas que ofrece el Tratado de Libre Comercio a la industria automotriz y las micro, pequeña y mediana empresas proveedoras de autopartes en un marco de desarrollo sustentable, aminorando riesgos y costos asociados a este desarrollo.

Derivado de las respuestas recibidas de los proveedores de Chrysler, Toluca, dentro de las siete variables de estudio, insisten en la rapidez de tiempo de ajuste que les da la empresa de estudio cuando hay cambio de un nuevo modelo, aunado a la importancia que tiene para ellos la colaboración y asistencia que les dan los ingenieros de la Chrysler para el logro de la eficiencia conjunta.

\section{Referencias}

Alchain, A. y A. Demsetz (1972). Production information costs, and economic organization. American Economic Association 62 (5): 777-795.

Aoki, Masaiko (1988). Information Incentives and Bargain in the Japanese Economy. New York: Cambridge University Press.

Asanuma, B. (1989). Manufacturer-supplier relation-ships un Japan and the concept $\mathrm{f}$ relation-specific Skill. Journal of the Japanese and International Economics 3: 1-30.

Baudry, V. (1995). L"economie des relations interentreprise. Paris: La Découverte, Collection Reperés (165).

Clark, K.B. y T. Fujimoto (1991). Product Development Performance. Harvard Business School Press, Boston, MA. 
Church, J. y W. Roger (2000). Industrial Organization A strategic Approach. EE.UU.: Mc. Graw-Hill.

De Banville, E. y J. J. Chanaron (1991). Vers un Systeme automobile européen. Paris: Económica.

Departamento de Ingeniería Industrial Chrysler, Planta de ensamble Toluca, 2011. Disponible en: http://www/amia.com.mx

Dyer Jeffrey. H. (1996). Specialized supplier networks as a source of competitive advantage: Evidence from the auto industry. Strategic Management Journal 17 (4): 271-292.

(1996) Does Governance matter? Keiretsu alliances and asset specificity as sources of Japanese competitive advantage. Organization Science 7(6): 649-666.

, W.G. Ouchi (1993). Japanese style business partnerships: Giving companies a competitive edge. Sloan Management Review 35 (1): 51-63.

Enright M. J. (1995). Organization and coordination in geographically concentrated Industries. En N. Lamoreaux and D. Raff (eds.). Coordination and Information: Historical Perspective on the Organization of Enterprise. University of Chicago. Press for the NBER, Chicago IL: 103-142.

Instituto Nacional de Estadistica, Geografia e Informatica (2006). La industria automotriz en México. Serie de estadísticas sectoriales.

Klein, B.,R. G. Crawford y A.A. Alchain (1978). Vertical integration, Appropriable rents, and the competitive contracting process. Journal of Law and Economics 21: 345-361.

Lundvall B.A. (1985). User Producer Relationship National System Innovation and Internationalization in B A. Ludvall ed. National System of Innovation Toward of Theory of Innovation an Interactive Learning, Printer Publisher, Londres, 1992. 
Nelson, R. y S.G. Winter (1982). An Evolutionary Theory of Economics Change. Harvard Massachusetts: Cambridge University Press.

Porter, M. (1991). Estrategia competitiva. Técnicas para el análisis de los sectores industriales y de la competencia. México: Cecsa.

(1992). Ventaja competitiva, creación y sostenimiento de un desempeño superior. Mexico: Cecsa.

Sako, M. (1992). Prices, Quality, Trust. Massachusetts: Cambridge University Press,

Shenkar, Y. L. (2004). International Business, United States of América. EE.UU.: John Willey \& sons.

Sidney G., W. (1993). On coase competence and the corporation. En O. E. Williamson y Sidney G. Winter. The nature of the firm.Origins, evolution and development. Inglaterra: Oxford University Press.

Shoemaker, P. y Amit (1994). Investment in strategic assets industry and firm level perspectives. En P. Shirivastava, A. Huff y J. Dutton (eds). Advances in Strategic Management. JAI Press, Grenwich, 10A.

Takahiro, F. (2001). The Japanese Automobile Parts Suppliers Systems the Triple of effective inter-firm routines. International Journal Automotive Technology and Management 1(1).

North, D.C. (1990), Institutional Change and Economic Performance. Cambridge University Press, Cambridge, UK.

Williamson, Q. E. (1983). Credible Commitments Using Hostages in Support exchange. Economic Review 73: 519-540.

Press.

(1985) The Economic Institutions of Capitalism. New York: Free

S. G. Winter (1993). The Nations of the Firm, Origins Evolution and Development. Oxford: University Press. 